\title{
A HIGHER-ORDER METHOD FOR NONLINEAR SINGULAR TWO-POINT BOUNDARY VALUE PROBLEMS
}

\author{
K. M. FURATI and M. A. EL-GEBEILY
}

Received 18 May 2001 and in revised form 3 October 2001

We present a finite difference method for a general class of nonlinear singular two-point boundary value problems. The order of convergence of the method for such a general class of problems is higher than the previous reported methods. The method yields a fourthorder convergence for the special case $p(x)=w(x)=x^{\alpha}, \alpha \geq 1$.

2000 Mathematics Subject Classification: 65L10.

1. Introduction. We consider the class of nonlinear singular two-point boundary value problems

$$
\begin{gathered}
-\frac{1}{w(x)}\left(p(x) y^{\prime}(x)\right)^{\prime}=g(x, y), \quad x \in(0,1), \\
\left(p y^{\prime}\right)\left(0^{+}\right)=0, \quad y(1)=0,
\end{gathered}
$$

under the following assumptions:

(A1) for $(x, y) \in[0,1] \times R$, the function $g(x, y)$ is continuous with continuous nonpositive derivative $g_{y}=\partial g / \partial y$;

(A2) $g(x)=g(x, y(x)) \in C^{m+1}[0,1]$, for some integer $m \geq 0$, whenever $y \in$ $C^{m+1}[0,1]$

(A3) $p^{-1}(x)=1 / p(x)$ is nonnegative and integrable on any compact subset of the interval $(0,1]$;

(A4) $w(x)$ is nonnegative and integrable on $[0,1]$;

(A5) $\int_{0}^{1}\left(\int_{t}^{1} p^{-1}(\tau) d \tau\right) w(t) d t<\infty$.

Under these assumptions, it was shown in [8] that the boundary conditions assumed here are possible and problem (1.1) has a unique solution $y(x)$ which is absolutely continuous on $[0,1]$.

Singular boundary value problems occur in many applications such as transport processes, thermal explosions, and electrohydrodynamics. (References are given in [4].)

Standard numerical methods exhibit loss of accuracy or even lack of convergence when applied to singular problems. (For more details please see [5, 9].)

There have been many numerical methods proposed for solving special cases of the nonlinear problem (1.1). In particular, for the case $w(x)=p(x)=x^{\alpha}, 0<\alpha<1$, Chawla and Katti [2] constructed a second-order three-point finite difference method. In [3], the method is extended to $\alpha \geq 1$. Later, Chawla et al. [4] constructed a fourthorder method for $\alpha \geq 1$. 
For the linear problem, Abu-Zaid and El-Gebeily [1] generalized the method in [3] to $w(x)=p(x)$. Recently, El-Gebeily and Abu-Zaid [7] relaxed this requirement and several other assumptions, however, the order of convergence of their scheme is at most 2.

We construct a higher-order method for the nonlinear problem (1.1). Unlike the previous treatment, this method is designed to work for general $p$ and $w$ which are not even required to be smooth. The higher order of convergence is obtained by approximating the function $g$ by a quadratic interpolating polynomial. This method is fourth order for the case $p(x)=w(x)=x^{\alpha}, \alpha \geq 1$, and at least third order for the class of problems considered in [1]. Quadrature methods can also be used to set up the integrals associated with this method. So, knowledge of the exact integrals is not necessary (except for those integrals that involve singularities).

We start by constructing the finite difference method. Then we analyze the error and find the rate of convergence. Finally, we present some numerical examples.

2. Exact discretization of the problem. In this section, we present the exact discretization of (1.1). We first introduce special sets of basis functions that we use for the discretization. Then we get a system of equations for the exact solution at the mesh points. Our working space is the space of continuous functions on the interval $[0,1]$.

Let $\pi=\left\{0=x_{0}<x_{1}<\cdots<x_{N}=1\right\}$ be a given partition of the interval [0,1]. We associate two sets of basis functions with the nodes $x_{1}, x_{2}, \ldots, x_{N-1}$ of this partition:

(1) the set $U_{1}, U_{2}, \ldots, U_{N-1}$ given by

$$
U_{1}(x)= \begin{cases}1, & x_{0} \leq x \leq x_{1} \\ \frac{\psi_{1}(x)}{\psi_{1}\left(x_{1}\right)}, & x_{1} \leq x \leq x_{2} \\ 0, & \text { otherwise }\end{cases}
$$

and for $2 \leq k \leq N-1$

$$
U_{k}(x)= \begin{cases}1-\frac{\psi_{k-1}(x)}{\psi_{k-1}\left(x_{k-1}\right)}, & x_{k-1} \leq x \leq x_{k}, \\ \frac{\psi_{k}(x)}{\psi_{k}\left(x_{k}\right)}, & x_{k} \leq x \leq x_{k+1}, \\ 0, & \text { otherwise, }\end{cases}
$$

where $\psi_{k}(x)=\int_{x}^{x_{k+1}} p^{-1}(t) d t$

(2) the local basis set $\ell_{i k}, i=k-1, k, k+1, k=1,2, \ldots, N-1$, of piecewise quadratic interpolating polynomials defined on each subinterval $I_{k}=\left[x_{k-1}, x_{k+1}\right]$ by

$$
\ell_{i k}(x)=\prod_{j=k-1, j \neq i}^{k+1} \frac{\left(x-x_{j}\right)}{\left(x_{j}-x_{i}\right)},
$$

and zero outside $I_{k}$.

We assume that

$$
\left\langle\ell_{i k}, w U_{k}\right\rangle \geq 0, \quad i=k-1, k, k+1, k=0, \ldots, N-1 .
$$


This assumption is satisfied by many standard problems. For example, one can show, by direct calculations, that this is the case when $w(x)=p(x)=x^{\alpha}$.

Let $\mathscr{P}_{k}$ be the projection

$$
\begin{gathered}
\mathscr{P}_{k}: C[0,1] \longrightarrow C\left(I_{k}\right), \\
\mathscr{P}_{k} f(x)=\sum_{j=-1}^{1} f_{k+j} \ell_{k+j, k}(x),
\end{gathered}
$$

where $f_{i}=f\left(x_{i}\right), i=0,1, \ldots, N-1$. If $f \in C^{3}\left(I_{k}\right)$, then

$$
\left(I-\mathscr{P}_{k}\right) f=\frac{f^{\prime \prime \prime}\left(\xi_{k}\right)}{3 !} \prod_{i=k-1}^{k+1}\left(x-x_{i}\right),
$$

for some $\xi_{k} \in I_{k}$.

By multiplying both sides of (1.1) by $U_{k}, k=1, \ldots, N-1$, and integrating over the interval $[0,1]$, we get

$$
\left\langle-\left(p y^{\prime}\right)^{\prime}, U_{k}\right\rangle=\left\langle g, w U_{k}\right\rangle
$$

where $\langle\cdot, \cdot\rangle$ is defined by

$$
\left\langle\alpha_{1}, \alpha_{2}\right\rangle=\int_{0}^{1} \alpha_{1}(x) \alpha_{2}(x) d x
$$

Note that $U_{k}$ is absolutely continuous on $[0,1]$ for $k=1, \ldots, N-1$. Therefore, if a function $y \in C[0,1]$ is such that $\left(p y^{\prime}\right)^{\prime}$ is integrable, then $\left\langle-\left(p y^{\prime}\right)^{\prime}, U_{k}\right\rangle=\left\langle p y^{\prime}, U_{k}^{\prime}\right\rangle$. If $y$ is a solution of the boundary value problem (1.1), then our assumptions on the functions $p, g, w$ imply that $\left(p y^{\prime}\right)^{\prime}$ is integrable. Hence, integration by parts is justified in our case.

It can be easily checked that the integrals in the left-hand side of system (2.7) take the more explicit form

$$
\begin{aligned}
-\left\langle\left(p y^{\prime}\right)^{\prime}, U_{1}\right\rangle & =-\frac{1}{\psi_{1}\left(x_{1}\right)} y_{2}+\frac{1}{\psi_{1}\left(x_{1}\right)} y_{1}, \\
-\left\langle\left(p y^{\prime}\right)^{\prime}, U_{k}\right\rangle & =\left\langle p y^{\prime}, U_{k}^{\prime}\right\rangle=\left\langle y^{\prime}, p U_{k}^{\prime}\right\rangle \\
& =-\frac{1}{\psi_{k}\left(x_{k}\right)} y_{k+1}+\left[\frac{1}{\psi_{k-1}\left(x_{k-1}\right)}+\frac{1}{\psi_{k}\left(x_{k}\right)}\right] y_{k}-\frac{1}{\psi_{k-1}\left(x_{k-1}\right)} y_{k-1},
\end{aligned}
$$

for $k=2, \ldots, N-1$, where $y_{i}=y\left(x_{i}\right)$. Also, by introducing the projections $\mathscr{P}_{k}$ into system (2.7), we get

$$
\left\langle p y^{\prime}, U_{k}^{\prime}\right\rangle=\left\langle\mathscr{P}_{k} g, w U_{k}\right\rangle+\left\langle\left(I-\mathscr{P}_{k}\right) g, w U_{k}\right\rangle
$$

with

$$
\left\langle\mathscr{P}_{k} g, w U_{k}\right\rangle=\sum_{i=k-1}^{k+1}\left\langle\ell_{i k}, w U_{k}\right\rangle g_{i}, \quad k=1, \ldots, N-1,
$$


where $g_{i}=g\left(x_{i}, y_{i}\right)$. Note that $g_{N}=g(1,0)$ while $g_{0}=g\left(0, y_{0}\right)$ involves the unknown value of the solution at $x=0$. We will deal with this difficulty later. It is known (see [8]), however, that $y_{0}$ exists and is finite under our assumptions.

In matrix form, system (2.7) can be written as

$$
T Y=L G(Y)+B(Y)+Q+R,
$$

where

$$
\begin{aligned}
Y & =\left[y_{1}, \ldots, y_{N-1}\right]^{t}, \\
G(Y) & =\left[g_{1}, \ldots, g_{N-1}\right]^{t}, \\
T & =\operatorname{tridiag}\left(t_{k}, \delta_{k}, t_{k+1}\right), \\
L & =\operatorname{tridiag}\left(l_{k-1}^{(k)}, l_{k}^{(k)}, l_{k+1}^{(k)}\right), \\
B(Y) & =\left[l_{0}^{(1)} g_{0}, 0, \ldots, 0\right]^{t}, \\
Q & =\left[0, \ldots, 0, l_{N}^{(N-1)} g_{N}\right]^{t}, \\
R & =\left[\left\langle\left(I-\mathscr{P}_{1}\right) g, w U_{1}\right\rangle, \ldots,\left\langle\left(I-\mathscr{P}_{N-1}\right) g, w U_{N-1}\right\rangle\right]^{t},
\end{aligned}
$$

with

$$
\begin{aligned}
t_{k+1} & =-\frac{1}{\psi_{k}\left(x_{k}\right)}, \quad k=1, \ldots,(N-2), \\
\delta_{1} & =-t_{2}, \quad \delta_{k}=-\left(t_{k+1}+t_{k}\right), \quad k=2, \ldots,(N-1), \\
l_{i}^{(k)} & =\left\langle\ell_{i k}, w U_{k}\right\rangle, \quad i=k-1, k, k+1, k=1, \ldots, N-1 .
\end{aligned}
$$

For $x \in\left[0, x_{1}\right]$, following the same derivation in [7], we can show the following identity:

$$
\begin{aligned}
y(x)= & y_{1}+\int_{0}^{x}\left(\int_{x}^{x_{1}} p^{-1}(\tau) d \tau\right) w(t) g(t) d t \\
& +\int_{x}^{x_{1}}\left(\int_{t}^{x_{1}} p^{-1}(\tau) d \tau\right) w(t) g(t) d t .
\end{aligned}
$$

In particular, at the singular point $x=0$, identity (2.15) reduces to

$$
y_{0}=y(0)=y_{1}+\int_{0}^{x_{1}}\left(\int_{t}^{x_{1}} p^{-1}(\tau) d \tau\right) w(t) g(t) d t,
$$

which can also be written as

$$
y_{0}-y_{1}=\left\langle g, w U_{0}^{+}\right\rangle,
$$

where

$$
U_{0}^{+}(x)= \begin{cases}\int_{x}^{x_{1}} p^{-1}(t) d t, & 0<x \leq x_{1} \\ 0, & \text { otherwise. }\end{cases}
$$


By expanding $g(x)$ about $\left(x, y_{1}\right)$, we get for $x \in\left(0, x_{1}\right)$,

$$
g(x)=g(x, y(x))=g\left(x, y_{1}\right)+g_{y}(x, y(\xi))\left(y-y_{1}\right),
$$

for some $\xi \in\left(x, x_{1}\right)$. Now, by using (2.17) and (2.19), we get the identity

$$
y_{0}=y_{1}+\left\langle g\left(\cdot, y_{1}\right), w U_{0}^{+}\right\rangle+\left\langle g_{y}(\cdot, y(\xi(\cdot)))\left(y-y_{1}\right), w U_{0}^{+}\right\rangle .
$$

3. Numerical method and error analysis. The setup carried out in Section 2 indicates that we intend to obtain a numerical method by truncating the remainder term $R$ in (2.12). However, one difficulty remains which is, how to obtain the value of $g_{0}$. To overcome this difficulty we notice that the basis function $U_{1}(x)$ is always unity on the interval $\left[0, x_{1}\right]$. This means that we may use an approximate value of $y_{1}, \bar{y}_{1}$, as an approximate value for $y_{0}$, and thus approximate $g_{0}=g\left(0, y_{0}\right)$ by $\bar{g}_{0}=g\left(0, \bar{y}_{1}\right)$. The fact that this approximation does not affect the overall order of the method remains to be shown.

Using this approximation of $g_{0}$ and truncating the remainder term $R$ in (2.12), we obtain a numerical method that determines an approximation $\bar{Y}=\left[\bar{y}_{1}, \ldots, \bar{y}_{N-1}\right]^{t}$ of $Y$ from

$$
T \bar{Y}=L G(\bar{Y})+B(\bar{Y})+Q .
$$

Then, the approximate value $\bar{y}_{0}$ of $y_{0}$ is calculated using

$$
\bar{y}_{0}=\bar{y}_{1}+\left\langle g\left(\cdot, \bar{y}_{1}\right), w U_{0}^{+}\right\rangle .
$$

For the error analysis, let $E=Y-\bar{Y}=\left[e_{1}, e_{2}, \ldots, e_{N-1}\right]^{t}$. Then, from (2.12) and (3.1), we get

$$
T E=L[G(Y)-G(\bar{Y})]+[B(Y)-B(\bar{Y})]+R .
$$

Using the integral form of the mean value theorem, we write

$$
G(Y)-G(\bar{Y})=\int_{0}^{1} G^{\prime}(t Y+(1-t) \bar{Y})(Y-\bar{Y}) d t=D E,
$$

where $G^{\prime}(Z)=\operatorname{diag}\left(g_{y}\left(x_{i}, z_{i}\right)\right)$ for $Z=\left[z_{1}, \ldots, z_{N-1}\right]^{t}$, and $D=\operatorname{diag}\left(d_{i}\right)$, where

$$
d_{i}=\int_{0}^{1} g_{y}\left(x_{i}, t y_{i}+(1-t) \bar{y}_{i}\right) d t
$$

For the term $B(Y)$, we write an error representation as follows: let $J=[1,0, \ldots, 0]^{t}$, then

$$
\begin{aligned}
B(Y)-B(\bar{Y}) & =l_{0}^{(1)}\left(g_{0}-\bar{g}_{0}\right) J=l_{0}^{(1)} J \int_{0}^{1} g_{y}\left(0, t y_{0}+(1-t) \bar{y}_{1}\right)\left(y_{0}-\bar{y}_{1}\right) d t \\
& =l_{0}^{(1)} d_{0}\left(y_{0}-\bar{y}_{1}\right) J=l_{0}^{(1)} d_{0} \epsilon_{1} J+l_{0}^{(1)} d_{0} e_{1} J \\
& =l_{0}^{(1)} d_{0} \epsilon_{1} J+F E,
\end{aligned}
$$


where $\epsilon_{1}=y_{0}-y_{1}, F=\operatorname{diag}(J) l_{0}^{(1)} d_{0}$, and

$$
d_{0}=\int_{0}^{1} g_{y}\left(0, t y_{0}+(1-t) \bar{y}_{1}\right) d t
$$

Hence, the error equation (3.3) can be rewritten as

$$
(T-L D-F) E=l_{0}^{(1)} d_{0} \epsilon_{1} J+R .
$$

Note that the entries of $D$ as well as $d_{0}$ are nonpositive since $g_{y} \leq 0$.

The matrix $T$ has the following properties:

(1) $T$ is tridiagonal and diagonally dominant,

(2) the diagonal elements are positive,

(3) $t_{k} t_{k+1}>0$, for $k=1, \ldots,(N-2)$.

It follows from these properties that $T$ is irreducible and thus $T$ is irreducibly diagonally dominant (see [10, pages 47-55]). Moreover, since the off-diagonal elements are nonpositive, $T$ is an $M$-matrix.

From (2.4), we have $L \geq 0$. This implies that $L D \leq 0$ and $F \leq 0$. Therefore, $T-L D-$ $F \geq T$. Also, $T-L D-F$ is an $M$-matrix. To see this, notice that

$$
\left|\frac{l_{k+1}^{(k)}}{t_{k+1}}\right|=\frac{\left\langle\ell_{i k}, w U_{k}\right\rangle}{1 / \psi_{k}\left(x_{k}\right)} \leq \int_{x_{k-1}}^{x_{k+1}} w(x)\left(\int_{x}^{x_{k+1}} p^{-1}(t) d t\right) d x,
$$

for $k=1,2, \ldots, N-1$, since $\left|\ell_{i k}\right| \leq 1$. It follows that for $h=\max _{0 \leq k \leq N-1}\left\{x_{k+1}-x_{k}\right\}$ sufficiently small, the off-diagonal elements of $T$ dominate the corresponding elements of $L D$. Hence, for sufficiently small $h, T-L D-F$ is an $M$-matrix and thus $(T-L D-F)^{-1} \leq T^{-1}$ (see [10]). Therefore, we have

$$
\|E\|_{\infty} \leq\left\|T^{-1}\left|l_{0}^{(1)} d_{0} \epsilon_{1} J+R\right|\right\|_{\infty}
$$

where the matrix $T^{-1}=\left(\tau_{k j}\right)$ is given by

$$
\tau_{k j}= \begin{cases}\int_{x_{j}}^{1} p^{-1}(x) d x, & k \leq j, \\ \int_{x_{k}}^{1} p^{-1}(x) d x, & k \geq j .\end{cases}
$$

It follows from (2.6) that for $1 \leq k \leq N-1,\left\|\left(I-\mathscr{P}_{k}\right) g\right\|_{\infty} \leq c h^{3}\left\|g^{\prime \prime \prime}\right\|_{\infty}$, and thus

$$
\left|\left\langle\left(I-\mathscr{P}_{k}\right) g, w U_{k}\right\rangle\right| \leq\left\|\left(I-\mathscr{P}_{k}\right) g\right\|_{\infty}\left\langle 1, w U_{k}\right\rangle \leq c h^{3}\left\|g^{\prime \prime \prime}\right\|_{\infty}\left\langle 1, w U_{k}\right\rangle
$$

Here $g^{\prime \prime \prime}(x)=\left(\partial^{3} / \partial x^{3}\right) g(x, y(x))$. 
Since the first row of $T^{-1}$ includes the largest element in each column, it follows from Lemma A.2 that

$$
\begin{aligned}
\left\|T^{-1}|R|\right\|_{\infty} & \leq c \sum_{k=1}^{N-1}\left(\left|\left\langle\left(I-\mathscr{P}_{k}\right) g, w U_{k}\right\rangle\right| \int_{x_{k}}^{1} p^{-1}(t) d t\right) \\
& \leq c h^{3}\left\|g^{\prime \prime \prime}\right\|_{\infty} \sum_{k=1}^{N-1}\left(\left\langle 1, w U_{k}\right\rangle \int_{x_{k}}^{1} p^{-1}(t) d t\right) \\
& =c h^{3}\left\|g^{\prime \prime \prime}\right\|_{\infty} \sum_{k=1}^{N-1}\left(\int_{x_{k-1}}^{x_{k+1}} w(t) U_{k}(t) d t \int_{x_{k}}^{1} p^{-1}(t) d t\right) \\
& \leq 2 c h^{3}\left\|g^{\prime \prime \prime}\right\|_{\infty} \sum_{k=1}^{N-1}\left(\int_{x_{k-1}}^{x_{k+1}}\left(\int_{t}^{1} p^{-1}(\tau) d \tau\right) w(t) d t\right) \\
& \leq 4 c h^{3}\left\|g^{\prime \prime \prime}\right\|_{\infty} \int_{0}^{1}\left(\int_{t}^{1} p^{-1}(\tau) d \tau\right) w(t) d t
\end{aligned}
$$

for some constant $c>0$. For the other term in the error equation (3.8), we have, from (2.20),

$$
\begin{aligned}
\left|\epsilon_{1}\right| & =\left|y_{0}-y_{1}\right| \\
& =\left|\left\langle g\left(\cdot, y_{1}\right), w U_{0}^{+}\right\rangle+\left\langle g_{y}(\cdot, y(\xi(\cdot)))\left(y-y_{1}\right), w U_{0}^{+}\right\rangle\right| \\
& \leq\|g\|_{\infty}\left\langle 1, w U_{0}^{+}\right\rangle+\left\|g_{y}\right\|_{\infty}\left\langle\left|y-y_{1}\right|, w U_{0}^{+}\right\rangle,
\end{aligned}
$$

and from (2.15) and Lemma A.1,

$$
\left|y-y_{1}\right| \leq\|g\|_{\infty}\left\langle 1, w U_{0}^{+}\right\rangle .
$$

Thus,

$$
\left|\epsilon_{1}\right| \leq\|g\|_{\infty}\left\langle 1, w U_{0}^{+}\right\rangle+\|g\|_{\infty}\left\|g_{y}\right\|_{\infty}\left\langle 1, w U_{0}^{+}\right\rangle^{2}=O\left(\left\langle 1, w U_{0}^{+}\right\rangle\right)
$$

Also from (3.7), $\left|d_{0}\right| \leq\left\|g_{y}\right\|_{\infty}$. Hence,

$$
\begin{aligned}
\left\|T^{-1}\left|l_{0}^{(1)} d_{0} \epsilon_{1} J\right|\right\|_{\infty} & =l_{0}^{(1)} \tau_{11}\left|d_{0} \epsilon_{1}\right| \\
& =O\left(l_{0}^{(1)} \tau_{11}\left\langle 1, w U_{0}^{+}\right\rangle\right) \\
& =O\left(\left\langle 1, w U_{0}^{+}\right\rangle \int_{0}^{x_{2}} w(t)\left(\int_{t}^{1} p^{-1}(\tau) d \tau\right) d t\right) \\
& =O\left(\left\langle 1, w U_{0}^{+}\right\rangle^{2}+\left[\int_{0}^{x_{2}} w(t)\left(\int_{t}^{1} p^{-1}(\tau) d \tau\right) d t\right]^{2}\right) \\
& =O\left(\left[\int_{0}^{x_{2}} w(t)\left(\int_{t}^{1} p^{-1}(\tau) d \tau\right) d t\right]^{2}\right) .
\end{aligned}
$$

The last equality holds because the first term in the previous equality is dominated by the second term. 
It follows from (3.13) and (3.17) that the order of the error in scheme (3.1) is

$$
\|E\|_{\infty}=O\left(h^{3}\right)+O\left(\left[\int_{0}^{2 h} w(t)\left(\int_{t}^{1} p^{-1}(\tau) d \tau\right) d t\right]^{2}\right) .
$$

As for the error in computing $y_{0}$, we have from (2.20), (3.2), and (3.15)

$$
\begin{aligned}
\left|y_{0}-\bar{y}_{0}\right| & =\left|\left(y_{1}-\bar{y}_{1}\right)+\left\langle g\left(\cdot, y_{1}\right)-g\left(\cdot, \bar{y}_{1}\right), w U_{0}^{+}\right\rangle+\left\langle g_{y}(\cdot, y(\xi(\cdot)))\left(y-y_{1}\right), w U_{0}^{+}\right\rangle\right| \\
& \leq\left|y_{1}-\bar{y}_{1}\right|+\left\|g_{y}\right\|_{\infty}\left|y_{1}-\bar{y}_{1}\right|\left\langle 1, w U_{0}^{+}\right\rangle+\left\|g_{y}\right\|_{\infty}\|g\|_{\infty}\left\langle 1, w U_{0}^{+}\right\rangle^{2} \\
& =O\left(\|E\|_{\infty}\right)+O\left(\left\langle 1, w U_{0}^{+}\right\rangle^{2}\right) \\
& =O\left(\|E\|_{\infty}\right)+O\left(\left[\int_{0}^{2 h} w(t)\left(\int_{t}^{1} p^{-1}(\tau) d \tau\right) d t\right]^{2}\right) .
\end{aligned}
$$

Thus we have proved the following theorem.

THEOREM 3.1. Under the assumptions (A1), (A2), (A3), (A4), (A5), and (2.4), the finite difference scheme (3.1) and (3.2) converges uniformly to the solution of (1.1) with order of convergence of at least

$$
O\left(h^{3}\right)+O\left(\left[\int_{0}^{2 h} w(t)\left(\int_{t}^{1} p^{-1}(\tau) d \tau\right) d t\right]^{2}\right) .
$$

REMARK 3.2. If the error term $\left\langle 1, w U_{0}^{+}\right\rangle^{2}$ is of less order than the error term $\left|y-\bar{y}_{1}\right|$, then more accuracy may be achieved by taking more terms in the expansion of $g$ about $y_{1}$. In principle, we get an error term of order $\left\langle 1, w U_{0}^{+}\right\rangle^{m+1}$, where $m$ is the number of derivatives taken in the Taylor series expansion of $g$.

REMARK 3.3. In the approximation (3.2), the exact integral $\left\langle g\left(\cdot, \bar{y}_{1}\right), w U_{0}^{+}\right\rangle$is computed. If this integral cannot be found in closed form or if it is too complicated, then it has to be computed numerically. This can be done by replacing $\left\langle g\left(\cdot, \bar{y}_{1}\right), w U_{0}^{+}\right\rangle$by

$$
g\left(x_{1}, \bar{y}_{1}\right)\left\langle 1, w U_{0}^{+}\right\rangle+\frac{\partial g}{\partial x}\left(x_{1}, \bar{y}_{1}\right)\left\langle\left(\cdot-x_{1}\right), w U_{0}^{+}\right\rangle .
$$

This results in the extra error term

$$
\left\langle\frac{\partial^{2}}{\partial x^{2}} g\left(\eta(\cdot), \bar{y}_{1}\right)\left(\cdot-x_{1}\right)^{2}, w U_{0}^{+}\right\rangle
$$

which is of order $h^{2}\left\langle 1, w U_{0}^{+}\right\rangle$.

REMARK 3.4. For the special case $p(x)=w(x)=x^{\alpha}, \alpha>1$, or if the differential equation is regular, the summation term in (3.13) is of order $h^{4}$. Also, the second term of (3.20) is of order $h^{4}$. So the method is fourth-order accurate as should be expected since our method and the method given in [4] are identical.

REMARK 3.5. In the more general situation $p(x)=O\left(x^{\alpha}\right), w(x)=O\left(x^{\beta}\right)$, at $x=0$, assumption (A5) is satisfied if and only if $\beta-\alpha+2>0$ and $\beta>-1$. If we let $\beta-\alpha+2=$ $\epsilon>0$, then the second term in Theorem 3.1 has the order $\min \left\{O\left(h^{2+2 \beta}\right), O\left(h^{2 \epsilon}\right)\right\}$. 
We end this section with a discussion of the question of existence and uniqueness of solutions of system (3.1). The result is best stated as a lemma.

LEMMA 3.6. System (3.1) has a unique solution.

Proof. The proof uses the theory of monotone operators (see [6]). We begin by showing that the matrix $T$ is positive definite. Let $V$ be the linear vector space generated by the basis functions $\left\{U_{i}\right\}_{i=1}^{N-1}$. Any $u \in V$ is absolutely continuous and satisfies the boundary condition $u(1)=0$. Therefore,

$$
|u(x)|=\left|\int_{x}^{1} u^{\prime}\right|=\left|\int_{x}^{1} p^{-1 / 2} p^{1 / 2} u^{\prime}\right| \leq\left(\int_{x}^{1} p^{-1}\right)^{1 / 2}\left(\int_{0}^{1} p\left(u^{\prime}\right)^{2}\right)^{1 / 2} .
$$

Hence,

$$
\int_{0}^{1} u^{2} w \leq\left(\int_{0}^{1} \int_{x}^{1} p^{-1} w\right) \int_{0}^{1} p\left(u^{\prime}\right)^{2}
$$

Now, writing $u=\sum y_{i} U_{i}$ and letting $Y=\left[y_{1}, y_{2}, \ldots, y_{N-1}\right]^{t}$, we can easily check that

$$
Y^{t} T Y=\int_{0}^{1} p u^{\prime 2}
$$

Therefore, by (3.24),

$$
Y^{t} T Y \geq\left(\int_{0}^{1} \int_{x}^{1} p^{-1} w\right)^{-1} \int_{0}^{1} u^{2} w>0
$$

This means that the minimum eigenvalue $\lambda_{m}$ of $T$ is positive and thus $T$ is positive definite.

Next, we show that the operator $T-G_{1}$ (where $G_{1}(Y)=L G(Y)+B(Y)+Q$ ) is strongly monotone. Let $X, Y \in \mathscr{R}^{N-1}$, then

$$
\begin{aligned}
(X-Y)^{t}\left[\left(T-G_{1}\right)(X)-\left(T-G_{1}\right)(Y)\right] \\
\quad \geq \lambda_{m}\|X-Y\|^{2}-(X-Y)^{t}\left[G_{1}(X)-G_{1}(Y)\right] \\
\quad=\lambda_{m}\|X-Y\|^{2}-(X-Y)^{t}\left(\int_{0}^{1} G_{1}^{\prime}(t X+(1-t) Y) d t\right)(X-Y) \\
\quad \geq \lambda_{m}\|X-Y\|^{2}
\end{aligned}
$$

since $G_{1}^{\prime} \leq 0$ and is diagonal. It follows from [6, Theorem 11.2] that $T-G_{1}$ is onto, that is, the equation $T(Y)-G_{1}(Y)=0$ has a solution. The uniqueness of this solution follows from the strong monotonicity of the operator $T-G_{1}$.

Since we do not have a contraction mapping principle here, Picard's iterations applied to (3.1) may not converge. We find the solution of (3.1) by Newton's method. For the implementation of Newton's method, we set $H(Y)=T Y-L G(Y)-B(Y)-Q$ and perform the usual iterations

$$
Y_{k+1}=Y_{k}-\left[H^{\prime}\left(Y_{k}\right)\right]^{-1} H\left(Y_{k}\right)
$$


with

$$
H^{\prime}(Y)=T-L G^{\prime}(Y)-l_{0}^{(1)} \frac{\partial g}{\partial y}\left(x_{0}, \bar{y}_{1}\right) F
$$

Standard theory for Newton's method and our assumptions (A1), (A2), (A3), (A4), and (A5) guarantee that the iterations (3.28) converge if the initial guess is sufficiently close to the true solution of (3.1).

4. Examples. In this section, we provide two numerical examples. The first example shows that with our scheme, we get a higher-order convergence than the scheme in [7] for a linear problem. In the second example, we solve a nonlinear problem.

EXAMPLE 4.1. Consider

$$
\begin{aligned}
p(x) & =\sin \left(\frac{\pi x}{2}\right), \\
w(x) & =1.0 \\
g(x, y) & =\frac{\pi^{2}}{2}\left\{\sin (\pi x)-\sin \left(\frac{\pi x}{2}\right) y\right\} .
\end{aligned}
$$

The exact solution is $y(x)=\cos (\pi x / 2)$ and the order of convergence, according to the scheme in [7], is $O(h \ln h)$. Numerical results using the new scheme are shown in Table 4.1. The results show that the order of the convergence of the relative error is about 3.6.

TABLE 4.1. The numerical results for Example 4.1.

\begin{tabular}{lc}
\hline$N$ & $\|Y-\bar{Y}\|_{\infty} /\|Y\|_{\infty}$ \\
\hline 16 & $6.7666 \times 10^{-5}$ \\
32 & $5.6235 \times 10^{-6}$ \\
64 & $4.4962 \times 10^{-7}$ \\
128 & $3.5246 \times 10^{-8}$ \\
\hline
\end{tabular}

EXAMPLE 4.2. Consider

$$
\begin{aligned}
p(x) & =\sin \left(\frac{\pi x}{2}\right) \\
w(x) & =1 \\
g(x, y) & =\left[\frac{\pi^{2}}{2} \sin \left(\frac{\pi x}{2}\right)+\cos \left(\frac{\pi x}{2}\right)\right] \cos \left(\frac{\pi x}{2}\right)-y^{2} .
\end{aligned}
$$

The exact solution is $y(x)=\cos (\pi x / 2)$. The results are shown in Table 4.2. The order of the convergence of the relative error is about 2.7. 
TABLE 4.2. The numerical results for Example 4.2.

\begin{tabular}{lc}
\hline$N$ & $\|Y-\bar{Y}\|_{\infty} /\|Y\|_{\infty}$ \\
\hline 16 & $3.1548 \times 10^{-4}$ \\
32 & $4.6984 \times 10^{-5}$ \\
64 & $7.0243 \times 10^{-6}$ \\
128 & $1.0413 \times 10^{-6}$ \\
\hline
\end{tabular}

Note that in both examples, the order predicted by Theorem 3.1 is at least $h^{2}(\ln h)^{2}$. Our numerical method achieved higher accuracy for both examples. For the special case $p(x)=w(x)=x^{\alpha}$, our method is identical to the method constructed in [4] and thus the order is $h^{4}$. Examples are presented in [4].

\section{Appendix}

\section{Some auxiliary lemmas}

LEMMA A.1. For $0 \leq a<x \leq b \leq 1$,

$$
\int_{a}^{x} w(t) d t \int_{x}^{b} p^{-1}(t) d t \leq \int_{a}^{x}\left(\int_{t}^{b} p^{-1}(\tau) d \tau\right) w(t) d t .
$$

Proof. The inequality follows from

$$
\begin{aligned}
\int_{a}^{x} w(t) d t \int_{x}^{b} p^{-1}(t) d t & =\int_{a}^{x}\left(\int_{x}^{b} p^{-1}(\tau) d \tau\right) w(t) d t \\
& \leq \int_{a}^{x}\left(\int_{t}^{b} p^{-1}(\tau) d \tau\right) w(t) d t
\end{aligned}
$$

since $p \geq 0$.

LEMMA A.2. Let

$$
\begin{gathered}
U_{x}^{-}(t)=\frac{\int_{x-\delta}^{t} p^{-1}(\tau) d \tau}{\int_{x-\delta}^{x} p^{-1}(\tau) d \tau}, \quad U_{x}^{+}(t)=\frac{\int_{t}^{x+\epsilon} p^{-1}(\tau) d \tau}{\int_{x}^{x+\epsilon} p^{-1}(\tau) d \tau}, \\
U_{x}(t)= \begin{cases}U_{x}^{-}(t), & x-\delta \leq t \leq x, \\
U_{x}^{+}(t), & x \leq t \leq x+\epsilon .\end{cases}
\end{gathered}
$$

Then,

$$
\int_{x-\delta}^{x+\epsilon} U_{x}(t) w(t) d t \int_{x}^{1} p^{-1}(t) d t \leq 2 \int_{x-\delta}^{x+\epsilon}\left(\int_{t}^{1} p^{-1}(\tau) d \tau\right) w(t) d t
$$

for $x-\delta \leq x \leq x+\epsilon$. 
Proof. Note that

$$
\begin{aligned}
\int_{x-\delta}^{x+\epsilon} U_{x}(t) w(t) d t \int_{x}^{1} p^{-1}(t) d t= & \int_{x-\delta}^{x} U_{x}^{-}(t) w(t) d t \int_{x}^{x+\epsilon} p^{-1}(t) d t \\
& +\int_{x}^{x+\epsilon} U_{x}^{+}(t) w(t) d t \int_{x}^{x+\epsilon} p^{-1}(t) d t \\
& +\int_{x-\delta}^{x+\epsilon} U_{x}(t) w(t) d t \int_{x+\epsilon}^{1} p^{-1}(t) d t
\end{aligned}
$$

For the first integral of (A.5), since $U_{x}^{-} \leq 1$, it follows from Lemma A.1 that

$$
\begin{aligned}
\int_{x-\delta}^{x} U_{x}^{-}(t) w(t) d t \int_{x}^{x+\epsilon} p^{-1}(t) d t & \leq \int_{x-\delta}^{x} w(t) d t \int_{x}^{x+\epsilon} p^{-1}(t) d t \\
& \leq \int_{x-\delta}^{x}\left(\int_{t}^{x+\epsilon} p^{-1}(\tau) d \tau\right) w(t) d t \\
& \leq \int_{x-\delta}^{x}\left(\int_{t}^{1} p^{-1}(\tau) d \tau\right) w(t) d t
\end{aligned}
$$

Similarly, for the third integral of (A.5), we have

$$
\begin{aligned}
\int_{x-\delta}^{x+\epsilon} U_{x}(t) w(t) d t \int_{x+\epsilon}^{1} p^{-1}(t) d t & \leq \int_{x-\delta}^{x+\epsilon} w(t) d t \int_{x+\epsilon}^{1} p^{-1}(t) d t \\
& \leq \int_{x-\delta}^{x+\epsilon}\left(\int_{t}^{1} p^{-1}(\tau) d \tau\right) w(t) d t
\end{aligned}
$$

For the second integral of (A.5), it follows from the definition of $U_{x}^{+}(t)$ that

$$
\begin{aligned}
\int_{x}^{x+\epsilon} U_{x}^{+}(t) w(t) d t \int_{x}^{x+\epsilon} p^{-1}(t) d t & =\int_{x}^{x+\epsilon}\left(\frac{\int_{t}^{x+\epsilon} p^{-1}(\tau) d \tau}{\int_{x}^{x+\epsilon} p^{-1}(\tau) d \tau}\right) w(t) d t \int_{x}^{x+\epsilon} p^{-1}(t) d t \\
& =\int_{x}^{x+\epsilon}\left(\int_{t}^{x+\epsilon} p^{-1}(\tau) d \tau\right) w(t) d t \\
& \leq \int_{x}^{x+\epsilon}\left(\int_{t}^{1} p^{-1}(\tau) d \tau\right) w(t) d t
\end{aligned}
$$

The results follow from (A.6), (A.7), and (A.8).

ACKNOWLEDGMENT. The authors are grateful for the financial support provided by King Fahd University of Petroleum and Minerals.

\section{REFERENCES}

[1] I. T. Abu-Zaid and M. A. El-Gebeily, A finite-difference method for the spectral approximation of a class of singular two-point boundary value problems, IMA J. Numer. Anal. 14 (1994), no. 4, 545-562.

[2] M. M. Chawla and C. P. Katti, Finite difference methods and their convergence for a class of singular two-point boundary value problems, Numer. Math. 39 (1982), no. 3, 341-350. 
[3] M. M. Chawla, S. McKee, and G. Shaw, Order $h^{2}$ method for a singular two-point boundary value problem, BIT 26 (1986), no. 3, 318-326.

[4] M. M. Chawla, R. Subramanian, and H. L. Sathi, A fourth order method for a singular two-point boundary value problem, BIT 28 (1988), no. 1, 88-97.

[5] P. G. Ciarlet, F. Natterer, and R. S. Varga, Numerical methods of high-order accuracy for singular nonlinear boundary value problems, Numer. Math. 15 (1970), 87-99.

[6] K. Deimling, Nonlinear Functional Analysis, Springer-Verlag, Berlin, 1985.

[7] M. A. El-Gebeily and I. T. Abu-Zaid, On a finite difference method for singular two-point boundary value problems, IMA J. Numer. Anal. 18 (1998), no. 2, 179-190.

[8] M. A. El-Gebeily, A. Boumenir, and M. B. M. Elgindi, Existence and uniqueness of solutions of a class of two-point singular nonlinear boundary value problems, J. Comput. Appl. Math. 46 (1993), no. 3, 345-355.

[9] P. Jamet, On the convergence of finite-difference approximations to one-dimensional singular boundary-value problems, Numer. Math. 14 (1969/1970), 355-378.

[10] J. M. Ortega and W. C. Rheinboldt, Iterative Solution of Nonlinear Equations in Several Variables, Academic Press, New York, 1970.

K. M. Furati: Department of Mathematical Sciences, King Fahd University of PETROlEum AND Minerals, DHAHran 31261, SAUDI ARABIA

E-mail address: kmfurati@kfupm. edu.sa

M. A. El-Gebeily: Department of Mathematical Sciences, King Fahd University of PETROLEUM AND Minerals, DHAHRAN 31261, SAUDI ARABIA

E-mail address: mgebei 7 y@kfupm. edu. sa 


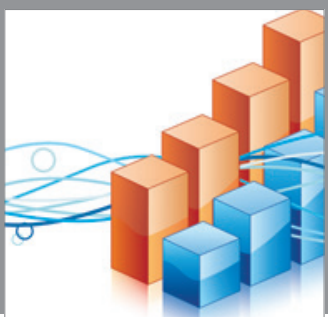

Advances in

Operations Research

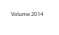

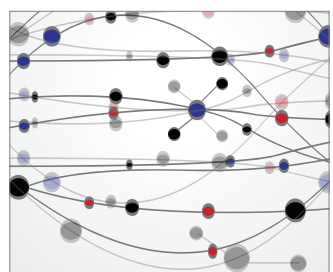

\section{The Scientific} World Journal
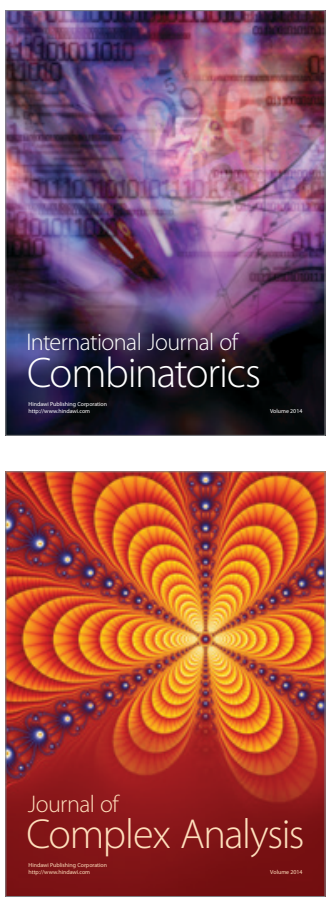

International Journal of

Mathematics and

Mathematical

Sciences
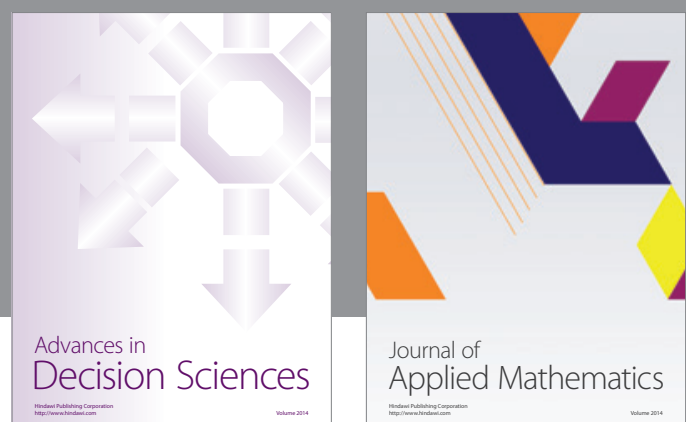

Journal of

Applied Mathematics
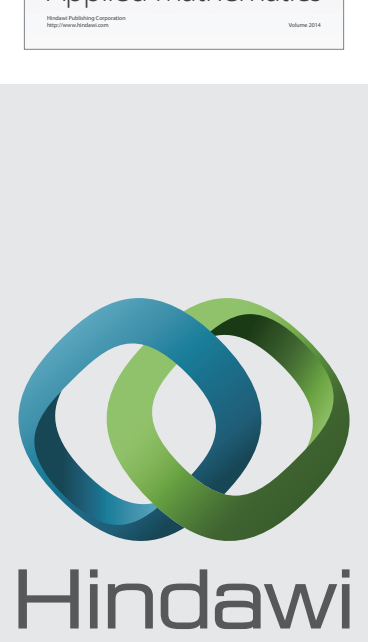

Submit your manuscripts at http://www.hindawi.com
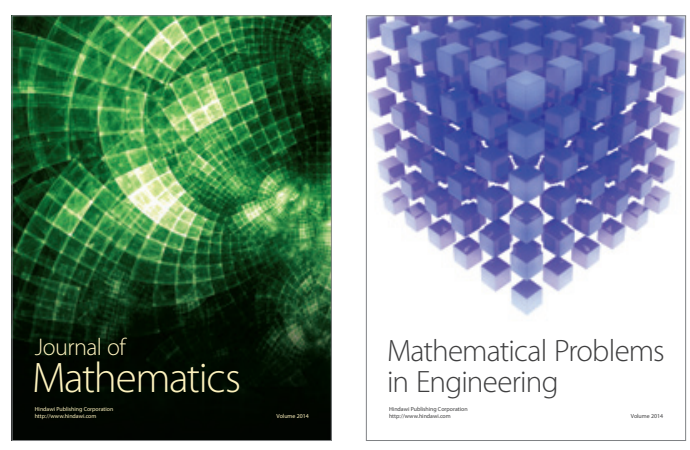

Mathematical Problems in Engineering
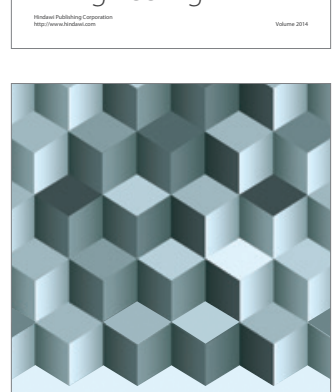

Journal of

Function Spaces
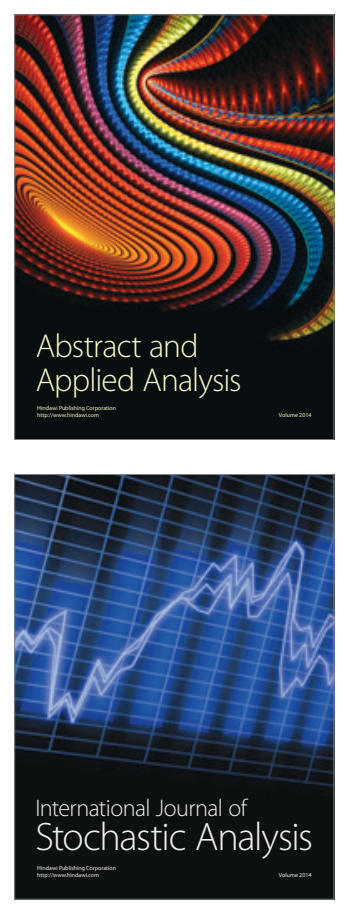

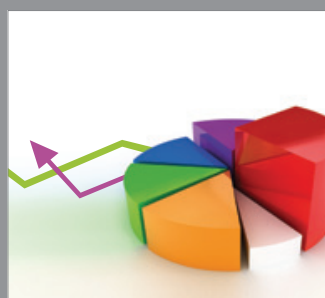

ournal of

Probability and Statistics

Promensencen
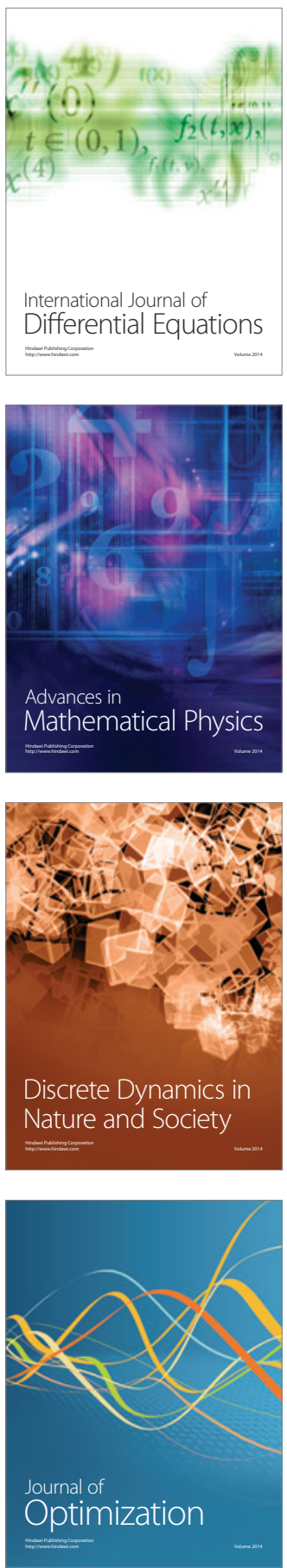